\title{
Frontal View Gait Recognition With Fusion of Depth Features From a Time of Flight Camera
}

\author{
Tengku Mohd Afendi Zulcaffle ${ }^{\circledR}$, Fatih Kurugollu ${ }^{\circledR}$, Senior Member, IEEE, \\ Danny Crookes ${ }^{\circledR}$, Senior Member, IEEE, Ahmed Bouridane ${ }^{\circledR}$, Senior Member, IEEE, \\ and Mohsen Farid, Senior Member, IEEE
}

\begin{abstract}
Frontal view gait recognition for people identification has been carried out using single RGB, stereo RGB, Kinect 1.0, and Doppler radar. However, existing methods based on these camera technologies suffer from several problems. Therefore, we propose a four-part method for frontal view gait recognition based on the fusion of multiple features acquired from a Time-of-Flight (ToF) camera. We have developed a gait data set captured by a ToF camera. The data set includes two sessions recorded seven months apart, with 46 and 33 subjects, respectively, each with six walks with five covariates. The four-part method includes: a new human silhouette extraction algorithm that reduces the multiple reflection problem experienced by ToF cameras; a frame selection method based on a new gait cycle detection algorithm; four new gait image representations; and a novel fusion classifier. Rigorous experiments are carried out to compare the proposed method with state-of-the-art methods. The results show distinct improvements over recognition rates for all covariates. The proposed method outperforms all major existing approaches for all covariates and results in $66.1 \%$ and $81.0 \%$ Rank 1 and Rank 5 recognition rates, respectively, in overall covariates, compared with a best state-of-the-art method performance of $35.7 \%$ and $57.7 \%$.
\end{abstract}

Index Terms-Gait recognition, frontal view, Time of Flight camera, fusion of features, depth gait data set.

\section{INTRODUCTION}

G AIT is the combination of posture and the way we move our whole body during the walking process [1]. It has been used as a discriminating feature in much recent research related to clinical analysis, gender classification, age estimation, forensics tools, and biometrics.

One interesting application in which gait features are used is biometrics. Among the earliest evidence for using gait

Manuscript received July 17, 2017; revised December 18, 2017, June 10, 2018, and August 1, 2018; accepted September 2, 2018. Date of publication September 17, 2018; date of current version November 8, 2018. The research work was sponsored through $\mathrm{PhD}$ fund by Ministry of Education, Malaysia (formerly known as Ministry of Higher Education, Malaysia). The associate editor coordinating the review of this manuscript and approving it for publication was Dr. Hafiz Malik. (Corresponding author: Tengku Mohd Afendi Zulcaffle.)

T. M. A. Zulcaffle was with the ECIT Institute, Queen's University Belfast, Belfast BT3 9DT, U.K. He is now with Universiti Malaysia Sarawak, Kota Samarahan, Sarawak 94300, Malaysia (e-mail: tzulcaffle01@qub.ac.uk).

F. Kurugollu and M. Farid are with the Department of Electronics, Computing and Mathematics, University of Derby, Derby DE22 1GB, U.K. (e-mail: f.kurugollu@derby.ac.uk; m.farid@derby.ac.uk).

D. Crookes is with the ECIT Institute, Queen's University Belfast, Belfast BT3 9DT, U.K. (e-mail: d.crookes@qub.ac.uk).

A. Bouridane is with the School of Computer and Information Sciences, Northumbria University, Newcastle upon Tyne NE1 8ST, U.K. (e-mail: ahmed.bouridane@northhumbria.ac.uk).

Color versions of one or more of the figures in this paper are available online at http://ieeexplore.ieee.org.

Digital Object Identifier 10.1109/TIFS.2018.2870594 as a biometric was the work of Murray et al. [2] and Johansson [3]. From a human anatomical point of view, Murray et al. suggested that gait is unique to an individual. Based on the experiments conducted by Johannson [3] and Stevenage et al. [4], they concluded that humans have the ability to identify individuals based on their gait. Unlike other biometrics such as fingerprint, finger veins, palmprint and palm veins, gait recognition can be used without direct contact with the sensing device. Unlike face and iris recognition, gait recognition does not require any specific postures or positions. It does not require the cooperation or even awareness of the individual under observation. Also, the gait is hard to conceal and difficult to disguise [1]. Gait features are perceivable at a distance, and only low resolution is required [5]-[7].

Although several approaches have been presented for gait recognition, most limit their attention to the lateral view, since this is considered to provide much more spatial and temporal information [8], [9]. However, this approach requires the camera to be placed at a certain height and distance, to capture full gait sequences. However, this is only applicable in outdoor or wide indoor spaces, and not in applications such as a secure narrow corridor. In such situations, frontal view gait recognition can be applied. Frontal view gait patterns can also be integrated with facial patterns to enhance biometric identification.

Early attempts at using frontal view gait recognition used a single RGB camera. Barnich and Van Droogenbroeck [10] proposed gait features derived from a set of rectangles fitting any closed silhouette in RGB video frames. However, the size of the rectangles has to be changed if a subject wears bigger clothes or high heel shoes. They managed to produce good results but tests were not carried out on the clothing and shoes covariates. Soriano et al. [8] and Balista et al. [11] applied Freeman Chain Code to the silhouette edge image. The method depends on high quality silhouette segmentation which is very difficult to achieve in a complex background. The frontal view gait recognition algorithm in [12] employs the $3 \mathrm{D}$ gait volume by placing the edge points of the silhouettes in a 3D space. Silhouette alignment is obtained by stacking the normalized bounding boxes over time. The major drawbacks of this method are that the edge points and stacking methods are very dependent on clothing, shoes, and carrying conditions. Soriano et al. [8] achieved $100 \%$ accuracy but the experiment only involved normal walk, with only 4 subjects who had to wear a special suit. Balista et al. [11] performed analysis 